Revista biomédica, 2017, 1, 28

Publicada en línea 1 febrero 2017 en http://revistabiomedica.mx/index.php/revbiomed/article/view/551

Revista Biomédica. http://www.revistabiomedica.mx

ISSN: 2007-8447

\title{
Bjomédica
}

Editorial

\section{Open Researcher and Contributor ID (ORCID)}

Gómez-Ontiveros Luis Vicente

Universidad Autónoma de Yucatán

\section{Autor para correspondencia}

Nombre: Luis Vicente Gómez Ontiveros

Institución: Universidad Autónoma de Yucatán

Departamento: Revista Biomédica, Centro de Investigaciones Regionales “Dr. Hideyo Noguchi”

Dirección: Avenida Itzaés \# 490 x calle 59, Colonia: Centro, C.P. 97000 Mérida, Yucatán, México

E-mail: luis.gomez@correo.uady.mx

Recibido: el 01 de diciembre de 2016 Aceptado para publicación: el 01 de diciembre de 2016

Copyright @ 2016 por autores (s) y Revista Biomédica.

Este trabajo esta licenciado bajo las atribuciones de la Creative Commons (CC BY).

http://creativecommons.org/licenses/by/4.0/

(c) (†) Open Access 
Gómez-Ontiveros L

En 2016, la revista biomédica realizó una actualización de su sitio web con el fin de lograr una mayor visibilidad de los artículos en el ciberespacio. Para lograr lo anterior se hizo uso del software especializado en revistas arbitradas, Open Journal Systems, que ofrece las condiciones necesarias para normalizar los datos de identificación tanto de la propia revista, como de cada uno de sus artículos.

Entre los elementos más importantes que se incorporaron con esta modernización se encuentran la introducción de metadatos a nivel de código web y descripción más amplia de los artículos y de los autores. En este contexto, una de las posibilidades que ofrece la revista biomédica, es la de agregar el Open Researcher and Contributor ID (ORCID), (Identificación de Investigador y Colaborador abierto), que es un número de identificación para los autores que permite la ubicación de todos sus artículos en las diferentes plataformas electrónicas (bases de datos, redes sociales, revistas, etc).

La aparición del ORCID tiene que ver con el aumento exponencial de la actividad editorial; cada día, se pueden encontrar más artículos en internet con formatos electrónicos como bases de datos o en sitios sin estructurar. Esto ha agudizado la dificultad para identificar a los autores complicando la, de por si alta, probabilidad de crear registros duplicados o ambiguos.

Como antecedente, en las bibliotecas han trabajado desde tiempo atrás en la solución de este problema creando registros de autoridad como una forma de identificación unívoca para personas y organizaciones, así como para crear las conexiones entre las diferentes formas de mencionar a un autor en los registros de los catálogos bibliográficos.

Asimismo, se impulsaron otras iniciativas creando diversos tipos de sistemas de identificación de autor, por ejemplo: Author Resolver, Inspire, IraLIS, RePEc, Scopus Author Identifier, ResearcherID, VIVO, entre otros otros, pero hasta ahora ninguno goza de una aplicación universal.

Varios especialistas han pensado en establecer un DAI, digital author identifier, algo semejante al DOI (Digital Object Identifier) que pudiera superar los inconvenientes derivados de hechos como la existencia de autores diferentes con el mismo nombre, firmas y afiliaciones cambiantes y procesos de indización automáticos e incapacidad de recuperar correctamente los problemas relacionados con la autoría; sin embargo, esto no había sido posible debido a la existencia de diversas barreras comerciales y técnicas. 
Es aquí donde destaca la iniciativa ORCID, cuya meta es establecer un registro abierto e independiente, asignando identificadores únicos enlazables a la producción científica del autor, independientemente del portal científico en el que este aparezca.

\section{Iniciativa ORCID}

ORCID, es una organización sin ánimo de lucro, que nació a finales del año 2009 a propuesta del Nature Publishing Group y de la empresa Thomson Reuters con el objetivo de crear un identificador de autores de publicaciones científicas, entendiendo por autor y publicaciones científicas en el término más amplio, o sea, todo creador de contenido científico y todo tipo de documento científico.

Como clave, ORCID, es un identificador compuesto por 16 dígitos, con estructura de url, basado en la norma ISO 27729:2012 (Information and documentation - International Standard Name Identifier - ISNI), que permite a los investigadores disponer de un código de autor persistente e inequívoco para distinguir claramente su producción científica" (1).

La plataforma de software de ORCID es Open Source, lo que le permite ser un proyecto global, abierto (en información y en software) e integrador (de universidades, agencias de financiación, editores) con contenido propio (2) que, además, ofrece la posibilidad de crear y mantener un registro único de investigadores, a través de un método transparente para articular las actividades de investigación. Es el único servicio que trata de asociar su propio identificador con otros identificadores de autor existentes; gracias a esta característica de integración los identificadores de autor específicos, como LATTES, Researcher-id o ID ArXiv Autor se puedan utilizar en paralelo con los identificadores universales (3). Es una iniciativa que busca ser transversal a través de todas las disciplinas, eliminando las fronteras existentes, ya sean estas geográficas, nacionales o institucionales.

La comunidad ORCID incluye a investigadores individuales, universidades, laboratorios nacionales, organizaciones de investigación comerciales, editores, agencias nacionales de ciencia, repositorios de datos, y asociaciones profesionales internacionales. Los principales editores que apoyan esta iniciativa incluyen a empresas como Nature Publishing Group, Elsevier, Hindawi, y varias instituciones de investigación tales como CERN, MIT, CalTech, etc.

Aun antes de que de ORCID estuviera en línea, más de 300 organizaciones ya habían registrado su apoyo, y 50 de ellas habían proporcionado recurso financiero para la puesta en marcha. 
Gómez-Ontiveros L

Desde su lanzamiento, varias organizaciones han estado integrando identificadores ORCID en sus flujos de trabajo de investigación, utilizando la interfaz de programación de aplicaciones (API) que ORCID ha puesto a la disposición de la comunidad.

Gracias a esta red de apoyo, la junta directiva de ORCID está compuesta por diferentes entidades como universidades, centros de investigación, editores, etcétera; lo que le proporciona un aspecto multidisciplinario e interinstitucional. Entre los miembros más destacados se encuentran lo siguientes: la Association for Computing Machinery (2016-2018): Bernie Rous, el CERN (20152017): Salvatore Mele, Chalmers University of Technology (2014-2016): Jonas Gilbert (2014-2015), Daniel Forsman (2015-2016), Cornell University (2015-2017): Simeon Warner, Crossref (20152017): Ed Pentz (Chair), Elsevier (2014-2016): Chris Shillum y una decena más de instituciones con amplio prestigio(4).

La obtención de un código ORCID, presenta ventajas para el investigador y la Institución a la que pertenece.

Para el investigador supone la obtención de un código único para sus publicaciones que las unifica y que además es interoperable con numerosas bases de datos.

Para la Institución, facilita el conocimiento de la actividad investigadora, la emisión de informes y la visibilidad de resultados.

ORCID puede incorporarse en varios sitios del investigador incluyendo, el perfil público, en la firma del correo electrónico, sus páginas web, las plataformas científicas en las que participe (ResearchGate, Academia.edu, Dialnet, etc.), como firma en el envío de manuscritos para su publicación, comunicaciones a congresos, como identificación en trabajos de peer-review y para la solicitud (o revisión) de proyectos de investigación (5).

Ustede puede crear una cuenta ORCID desde https://orcid.org/, los pasos a seguir para el registro son muy sencillos solo hay que llenar unos cuantos datos que incluyen su nombre, apellido, correo electrónico y password.

Finamente le recomendamos ampliamente el uso del identificador ORCID por todos los beneficios que aporta al investigador, incluyendo la garantía de que sus trabajos siempre serán recuperados por los usuarios y acreditados a su legitimo autor. 


\section{Referencias}

1. $\quad$ ORCID | Apoyo a la Investigación en Biblioteca [en línea]. 2016 Noviembre [Fecha de acceso 30 Noviembre 2016]. Disponible en: http://www2.ual.es/apoyoinv/orcid-open-researcher-and-contributor-id/

2. García-Gómez C. ORCID: un sistema global para la identificación de investigadores. El Prof Inf. 2012 Jan $1 ; 21(2): 210-212$.

3. Un análisis de los principales sistemas de identificación y perfil para el personal investigador - Dialnet [en línea]. 2016 Noviembre [Fecha de acceso 30 Noviembre 2016]. Disponible en: https://dialnet.unirioja.es/servlet/articulo?codigo $=3921021$

4. Comunidad ORCID | ORCID [en línea]. 2016 Diciembre [Fecha de acceso 1 Diciembre 2016]. Disponible en: http://orcid.org/about/community

5. Haak LL, Fenner M, Paglione L, Pentz E, Ratner H. ORCID: a system to uniquely identify researchers. Learn Publ. 2012 Oct 1;25(4):259-64. 